\title{
Role of electrocardiograms in assessment of severity and analysis of the characteristics of ST elevation in acute myocarditis: A two-centre study
}

\author{
JIAOZHEN CHEN ${ }^{1}$, SHOUQUAN CHEN ${ }^{2},{\text { ZHANGPING } \text { LI }^{2} \text {, PEISEN ZHOU }}^{2}$, WEIJIA HUANG ${ }^{2}$, \\ HETE WANG ${ }^{1}$, JINCUN SHI ${ }^{3}$, YUNCHAO NI ${ }^{2}$, LILI LIN ${ }^{1}$ and YUANLI LEI ${ }^{2}$ \\ ${ }^{1}$ Department of Electrocardiogram, Wenzhou People's Hospital; ${ }^{2}$ Department of Emergency Medicine, \\ The First Affiliated Hospital of Wenzhou Medical University; ${ }^{3}$ Department of Critical Care Medicine, \\ Wenzhou Central Hospital, Wenzhou, Zhejiang 325000, P.R. China
}

Received January 4, 2020; Accepted July 22, 2020

DOI: $10.3892 /$ etm.2020.9148

\begin{abstract}
Acute myocarditis is a severe disease with a high mortality rate and various dynamic changes visible on electrocardiograms (ECGs). The purpose of the present study was to investigate ECG findings of patients with acute myocarditis, ECG findings associated with fulminant myocarditis (FM) and the characteristics of ST elevation on admission. A retrospective analysis of 1,814 ECGs of 274 consecutive patients with acute myocarditis aged $\geq 13$ years, who were hospitalized in two centres between August 2007 and November 2019, was performed. A total of 251 patients with myocarditis $(91.6 \%)$ presented with ECG abnormalities. The most common ECG findings were T-wave inversion and ST elevation. Univariate logistic regression analysis demonstrated that 12 ECG findings were associated with FM. Multivariate regression analysis revealed that the independent predictive factors for FM included ventricular tachycardia, high-degree atrioventricular block, sinus tachycardia, low voltage and QRS duration of $\geq 120 \mathrm{msec}$ (all $\mathrm{P}<0.05$ ). A total of 112 cases displayed ST elevation at admission. Of these, ST elevation without T-wave inversion $(n=87)$ was associated with a shorter duration of cardiac symptoms (1.5 vs. 3.1 days; $\mathrm{P}<0.001)$ compared with ST elevation with T-wave inversion $(n=25)$. Of the aforementioned 87 patients, $71(81.6 \%)$ presented with T-wave inversion at the hospital. The median time from the onset of cardiac symptoms to T-wave inversion was 4.0 days. In conclusion, patients with acute myocarditis exhibited various dynamic changes on ECG. Thus, ECGs should be widely used for the assessment of severity and the characteristics of ST elevation on admission.
\end{abstract}

Correspondence to: Dr Yuanli Lei, Department of Emergency Medicine, The First Affiliated Hospital of Wenzhou Medical University, 1 West Fanhai Road, Wenzhou, Zhejiang 325000, P.R. China

E-mail: 191248120@qq.com

Key words: acute myocarditis, electrocardiogram, fulminant, cardiac arrest, ST elevation, atrioventricular block

\section{Introduction}

Acute myocarditis, a common, easily misdiagnosed disease with a high mortality rate, is defined as an inflammatory infiltrate of the myocardium that results from viral infections, autoimmune diseases or cardiotoxic agents $(1,2)$. This inflammation may lead to acute heart failure, chest pain and electrocardiographic abnormalities (3).

Various electrocardiogram (ECG) findings have been reported in patients with myocarditis (4-6). The common ECG findings are ST-T wave changes, Q waves, QT interval prolongation, QRS prolongation, low voltage, atrioventricular block (AVB), bundle branch block (BBB) and ventricular tachycardia (VT) (4-7). However, studies on this topic are currently limited, focusing on abnormal ECG findings and their rate of abundance in patients with acute myocarditis $(4,8)$. Furthermore, most of the aforementioned ECG findings (such as VT, AVB, QRS prolongation and low voltage) are associated with the severity of myocarditis $(5,7,8)$. However, these studies are mainly on certain ECG changes for predicting outcomes, and the associations between ECG findings and the severity are simply mentioned $(5,7)$. Fulminant myocarditis (FM) is an acute and severe form of myocarditis (9). FM causes acute hemodynamic instability which may prove fatal (10). FM is associated with a wide variety of serious arrhythmias such as VF and AVB (11). AVB is an important ECG aberration in patients with myocarditis, which is associated with the severity of the disease (7). However, the association between AVB and CA remains to be fully elucidated. Furthermore, ST-T wave changes, including T-wave inversion and ST segment elevation, are the most common and the most widely studied ECG changes in myocarditis $(2,5)$. Most patients with myocarditis present with ST elevation mimicking ECG alterations caused by AMI. There is currently a shortage of studies on the characteristics of ST elevation in a large cohort of patients with myocarditis $(1,4)$. Furthermore, it has been indicated that certain patients with myocarditis with ST elevation progress further to T-wave inversion (11). However, only a small number of studies with small sample of patients present data regarding the prognosis of such patients $(1,4)$. 
Although ECGs may be easily recorded, the ECG results of only a small sample of patients with abnormal ECG findings in myocarditis are currently available $(1,4,5,12)$. The ECG abnormalities, their association with severity and the characteristics of ST elevation remain to be fully investigated in a large cohort of patients with myocarditis. The purpose of the present study was to identify the role of and draw attention to the use of ECG in myocarditis. The present study specifically sought to identify specific ECG findings associated with FM and assess the characteristics of ST elevation in these patients on admission through a review of patient databases at two centres in China.

\section{Materials and methods}

Study population. The present study was a two-centre retrospective review of all patients aged $\geq 13$ years who were hospitalized with a diagnosis of acute myocarditis between August 2007 and November 2019. The medical records were reviewed with permission from ethics committee of The First Affiliated Hospital of Wenzhou Medical University (approval no. KY2020-103) and Wenzhou People's Hospital (approval no. 2020-96). On the basis of the diagnostic criteria of European Society of Cardiology (2) and Japanese Circulation Society (10), the diagnosis of acute myocarditis was confirmed by a recent history of gastrointestinal/upper respiratory tract infection and/or complaints of cardiac symptoms and increasing myocardiocytolysis markers and/or presentation with a new abnormality on the 12-lead ECG, combined with at least one of the following: i) Active or borderline biopsy according to the Dallas criteria (13); ii) positive infectious origin of ventricular dysfunction; iii) delayed enhancement on cardiac MRI consistent with myocarditis; or iv) serological tests, ECGs, ultrasonic cardiogram (UCG), coronary angiography and ventriculography to exclude acute myocardial infarction (AMI), stress cardiomyopathy, congenital heart disease, myocarditis secondary to sepsis, valve disease, hyperthyroidism, autoimmune disease and rheumatic fever. The exclusion criteria were as follows: i) Incomplete data; ii) a history of congenital heart disease, cardiomyopathy or arrhythmias; iii) myocarditis not being the primary diagnosis for an admission; and iv) absence of cardiac symptoms. Cardiac symptoms were considered to comprise chest pain, chest discomfort, syncope, dyspnoea, palpitations, shock, seizure and cyanosis (10).

Diagnosis of FM and patient management. In patients with acute myocarditis, a diagnosis of FM was determined upon identification of one or more of the following: Haemodynamic instability due to cardiogenic shock or arrhythmia; left ventricular dysfunction and low cardiac output syndrome requiring inotropes or mechanical circulatory support; mechanical ventilation; and/or cardiac arrest (CA) $(9,14,15)$. In the present study, FM was diagnosed in 77 patients $(28.1 \%)$ on the basis of the aforementioned criteria, whereas the remaining 197 patients (71.9\%) were classified as non-FM. The treatment plan was established according to the guidelines for different stages of the disease and the patient's condition $(2,10)$.
ECG data collection and analysis. Standard hospital procedures were followed. When the patient suspected to have myocarditis was admitted to the hospital, a standard 12-lead ECG was performed immediately and 24-h ECG monitoring was performed in parallel. If the ECG exhibited any abnormality in the right ventricular or posterior wall of the left ventricle, an 18-lead ECG was added (16). If the ECG indicated arrhythmia, a 1-min ECG was added. If the condition of the patient had changed or the 24-h ECG monitoring indicated changes in any ECG parameter, the standard 12-lead/18-lead/1-min ECG was again performed immediately. ECG changes, including ST elevation or depression, abnormal $\mathrm{T}$ waves, low voltage, abnormal Q waves, QTc prolongation, AVB, BBB, premature beats, atrial fibrillation, supraventricular tachycardia and VT were recorded and compared with the ECG abnormality onsets. Once ECGs were performed, the rhythm, heart rate, PR interval, QRS duration, QTc interval and ST segment/T-wave changes of all ECGs obtained during the hospital stay were analyzed independently by two electrophysiologists (JC, LL) to identify any abnormalities without any further delay. The discrepancies between the evaluation results determined by the electrophysiologists were solved by discussion and a vote (ZL, HW).

Electrocardiographic definitions. ST elevation was defined as an elevation of the $\mathrm{J}$ point in at least two contiguous leads of $\geq 0.2 \mathrm{mV}$ in chest leads V2-V3, and/or of $\geq 0.1 \mathrm{mV}$ in other leads or limb leads with or without reciprocal ST depression $(1,17,18)$. ST depression was defined as ST segment depression $\geq 0.2 \mathrm{mV}$ in at least two leads, not concurrent with ST elevation (5). Abnormal $\mathrm{T}$ waves included flat, inverted or diphasic $\mathrm{T}$ waves. T-wave inversion was defined as a negative $\mathrm{T}$ wave $\geq 0.1 \mathrm{mV}$ in at least two of the following leads: I, II, avL and V2-V6 (18). Any $\mathrm{Q}$ waves $>40 \mathrm{msec}$ were considered abnormal (4). Low voltage was defined as voltages $\leq 0.5 \mathrm{mV}$ in all limb leads or $\leq 1.0 \mathrm{mV}$ in all precordial leads (5). The QT interval was corrected using the Bazett formula $(\mathrm{QTc}=\mathrm{QT} / \sqrt{ } \mathrm{RR})(8)$. QTc prolongation was defined as a QTc duration $\geq 440 \mathrm{msec}(8)$. QRS prolongation was defined as a QRS duration $\geq 120 \mathrm{msec}$ in sinus rhythm (8). VT was diagnosed when six or more consecutive premature ventricular contractions occurred (4). Bradyarrhythmias/tachyarrhythmias and conduction disturbances, including premature beats, sinus tachycardia or bradycardia, supraventricular tachycardia, AVB and new $\mathrm{BBB}$, were identified by referring to the ECG and 1-min ECG. $A V B$ included AVBs that were non-advanced (first-degree AVB, non-advanced second-degree AVB) or high-degree (second-degree Mobitz type II AVB and complete AVB) (7).

Statistical analysis. Data were analyzed using the statistical program SPSS version 18.0 (SPSS, Inc.). Numerical data are expressed as the mean \pm standard deviation and categorical data are expressed as n (\%). Student's t-test was used for numerical variables and Pearson's $\chi^{2}$ test for categorical variables. A $\chi^{2}$ test was used to evaluate and compare the abnormal ECG findings between the FM and non-FM groups. Multivariate (unconditional) logistic regression analysis was performed to further evaluate ECG findings associated with FM and was performed for significant variables $(\mathrm{P} \leq 0.05)$. A main effect model was used in logistic regression analysis. 
The Mann-Whitney U test was used to compare the duration of cardiac symptoms prior to admission for ST elevation at admission. A two-sided $\mathrm{P}<0.05$ was considered to indicate statistical significance.

\section{Results}

Basic data. A total of 274 cases of acute myocarditis (234 cases from The First Affiliated Hospital of Wenzhou Medical University and 40 cases from Wenzhou People's Hospital), including 186 males and 88 females, were included in the present study. The mean age of the patients was $36.97 \pm 16.22$ years (range, 13-93 years; Table SI). Among all cases, 77 were diagnosed with FM, 71 cases experienced cardiogenic shock, 27 cases experienced CA and 14 died. The median duration of cardiac symptoms prior to admission was 2 days (range, 1-3 days) and the median length of hospital stay was 8 days (range, 5-12 days). All 1,814 ECGs were collected and analyzed (not including the initial ECG recording during CA). A mean of 4 ECGs (range, 2-9 ECGs) was obtained per patient, with the greatest number of ECGs in a patient being 62 .

ECG findings and their association with FM. Among the 274 cases of myocarditis, 251 cases $(91.6 \%)$ presented with abnormal ECG findings. There were at least two abnormal findings in each of the 192 patients, with the greatest number of findings in a patient being 10. The most common ECG findings were abnormal $\mathrm{T}$ waves $(57.7 \%)$ /T-wave inversion $(48.2 \%)$ and ST elevation (44.2\%) (Table I). A comparison of general data for FM and non-FM are listed in Table SI (all P>0.05). Compared with the non-FM group, the FM group had significantly higher values for 12 ECG parameters, which are listed in Table II (all $\mathrm{P}<0.05)$. Multivariate logistic regression analysis revealed that the independent predictive factors associated with FM were VT, high-degree AVB, sinus tachycardia, low voltage and $\mathrm{QRS}$ duration of $\geq 120 \mathrm{msec}$ (all $\mathrm{P}<0.05$; Table III).

The incidence of $C A$ in different types of $A V B$ and the initial ECG recorded during $C A$. As presented in Fig. 1, 38 cases presented with AVB, including 6 cases that had both non-advanced AVB and high-degree AVB (of the aforementioned 6 patients, 1 case with CA). A total of 12 cases had non-advanced AVB, including 2 cases with $\mathrm{CA}$; and 32 cases had high-degree AVB, including 9 cases with $\mathrm{CA}$. The incidence rate of CA (7.2 vs. $26.3 \%$; $\mathrm{P}=0.001)$ was different between the non-AVB $(n=236)$ and AVB groups $(n=38)$; and the incidence of CA (7.2 vs. 28.1\%; $\mathrm{P}=0.001)$ was different between non-AVB $(n=236)$ and high-degree AVB $(n=32)$ patients (Table SII). A total of 27 patients experienced CA. The initial rhythm of 21 patients $(77.8 \%)$ was a shockable rhythm [ventricular fibrillation (VF) or pulseless VT]. The initial rhythm of 5 patients $(18.5 \%)$ was a non-shockable rhythm, including 3 cases of pulseless electrical activity (11.1\%) and 2 cases of asystole (7.4\%). The initial rhythm of 1 patient was unknown (Fig. S1).

Investigation of patients presenting with ST elevation at admission. Of the 121 cases with ST elevation (of which 5 cases had reciprocal ST depression), 112 cases displayed ST elevation at admission (of which 3 cases had reciprocal
Table I. Abnormal findings on electrocardiograms in patients with acute myocarditis.

\begin{tabular}{lc}
\hline Variable & $\mathrm{N}(\%)$ \\
\hline Abnormal T waves & $158(57.7)$ \\
T-wave inversion & $132(48.2)$ \\
ST elevation & $121(44.2)$ \\
Sinus tachycardia & $72(26.3)$ \\
QRS duration of $\geq 120$ msec & $68(24.8)$ \\
Right bundle branch block & $46(16.8)$ \\
Left bundle branch block & $17(6.2)$ \\
Intraventricular block & $11(4.0)$ \\
QTc prolongation & $61(22.3)$ \\
Abnormal Q waves & $48(17.5)$ \\
Low voltage & $44(16.1)$ \\
High-degree atrioventricular block & $32(11.7)$ \\
Reduced R-wave height & $28(10.2)$ \\
Ventricular/junctional escape beats & $26(9.5)$ \\
Sinus bradycardia & $24(8.8)$ \\
Ventricular premature beats & $23(8.4)$ \\
ST depression & $18(6.6)$ \\
Ventricular tachycardia & $17(6.2)$ \\
Atrial premature beats & $13(4.7)$ \\
Sinus arrhythmia & $13(4.7)$ \\
Non-advanced atrioventricular block & $12(4.4)$ \\
Paroxysmal rapid atrial fibrillation & $9(3.3)$ \\
Accelerated idioventricular/idiojunctional rhythm & $8(2.9)$ \\
Interference atrioventricular dissociation & $5(1.8)$ \\
Paroxysmal supraventricular tachycardia & $3(1.1)$ \\
\hline &
\end{tabular}

ST depression). Of these, 87 had ST elevation without T-wave inversion and the median duration of cardiac symptoms prior to admission was 1.5 days (range, 0.1-6.0 days). A total of 25 patients had ST elevation with T-wave inversion and the median duration of cardiac symptoms prior to admission was 3.1 days (range, 1.0-7.0 days). ST elevation without T-wave inversion was associated with a shorter duration of cardiac symptoms (median value, 1.5 vs. 3.1 days; $\mathrm{P}<0.001$ ) compared with ST elevation with $\mathrm{T}$ wave inversion (Fig. 2; Table SIII). Of the 87 patients with ST elevation without T-wave inversion at admission, 71 patients $(81.6 \%)$ had T-wave inversions at the hospital. The median time from the onset of cardiac symptoms to T-wave inversion was 4.0 days (range, 1.0-16.0 days; Fig. 3). The ECG changes in the evolution from ST elevation at admission to T-wave inversion of a 14-year-old male patient are presented in Fig. 4.

\section{Discussion}

Acute myocarditis, a common, easily misdiagnosed disease with a high mortality rate, is defined as an inflammatory infiltrate of the myocardium with necrosis and degeneration of adjacent myocytes as a result of viral infections, autoimmune diseases or cardiotoxic agents $(1,2)$. Since myocarditis has a highly variable clinical presentation, ranging from mild symptoms to severe heart 
Table II. Comparison of the significant electrocardiogram findings for FM and non-FM.

\begin{tabular}{|c|c|c|c|c|c|}
\hline Variable & $\mathrm{N}$ & FM $77(28.1 \%)$ & Non-FM 197 (71.9\%) & $\chi^{2}$ & P-value \\
\hline ST elevation & 121 & $42(54.5)$ & $79(40.1)$ & 4.684 & 0.030 \\
\hline Sinus tachycardia & 72 & $35(45.5)$ & $37(18.8)$ & 20.331 & $<0.001$ \\
\hline QRS duration of $\geq 120 \mathrm{msec}$ & 68 & $36(46.8)$ & $32(16.2)$ & 27.619 & $<0.001$ \\
\hline Abnormal Q waves & 48 & $23(29.9)$ & $25(12.7)$ & 11.308 & 0.001 \\
\hline Low voltage & 44 & $21(27.3)$ & $23(11.7)$ & 9.992 & 0.002 \\
\hline High-degree atrioventricular block & 32 & $25(32.5)$ & $7(3.6)$ & 44.871 & $<0.001$ \\
\hline Reduced R-wave height & 28 & $14(18.2)$ & $14(7.1)$ & 7.401 & 0.007 \\
\hline Ventricular/junctional escape beats & 26 & $15(19.5)$ & $11(5.6)$ & 12.448 & $<0.001$ \\
\hline Ventricular premature beats & 23 & $11(14.3)$ & $12(6.1)$ & 4.834 & 0.028 \\
\hline Ventricular tachycardia & 17 & $16(20.8)$ & $1(0.5)$ & 35.687 & $<0.001$ \\
\hline Non-advanced atrioventricular block & 12 & $8(10.4)$ & $4(2.0)$ & 7.349 & 0.007 \\
\hline Paroxysmal rapid atrial fibrillation & 9 & $7(9.1)$ & $2(1.0)$ & 8.965 & 0.003 \\
\hline
\end{tabular}

FM, fulminant myocarditis.

Table III. Multivariate regression analysis of factors associated with fulminant myocarditis.

\begin{tabular}{lccrrrr}
\hline Variable & $\beta$ & Std. Error & Wald & P-value & OR & $95.0 \%$ CI for OR \\
\hline Ventricular tachycardia & 3.213 & 1.122 & 8.199 & 0.004 & 24.859 & $2.756-224.236$ \\
High-degree atrioventricular block & 3.142 & 0.714 & 19.379 & $<0.001$ & 23.157 & $5.716-93.816$ \\
Sinus tachycardia & 1.288 & 0.379 & 11.576 & 0.001 & 3.627 & $1.727-7.618$ \\
Low voltage & 1.089 & 0.433 & 6.318 & 0.012 & 2.972 & $1.271-6.951$ \\
QRS duration of $\geq 120 \mathrm{msec}$ & 0.904 & 0.393 & 5.279 & 0.022 & 2.468 & $1.142-5.336$
\end{tabular}

OR, odds ratio; Std., standard.

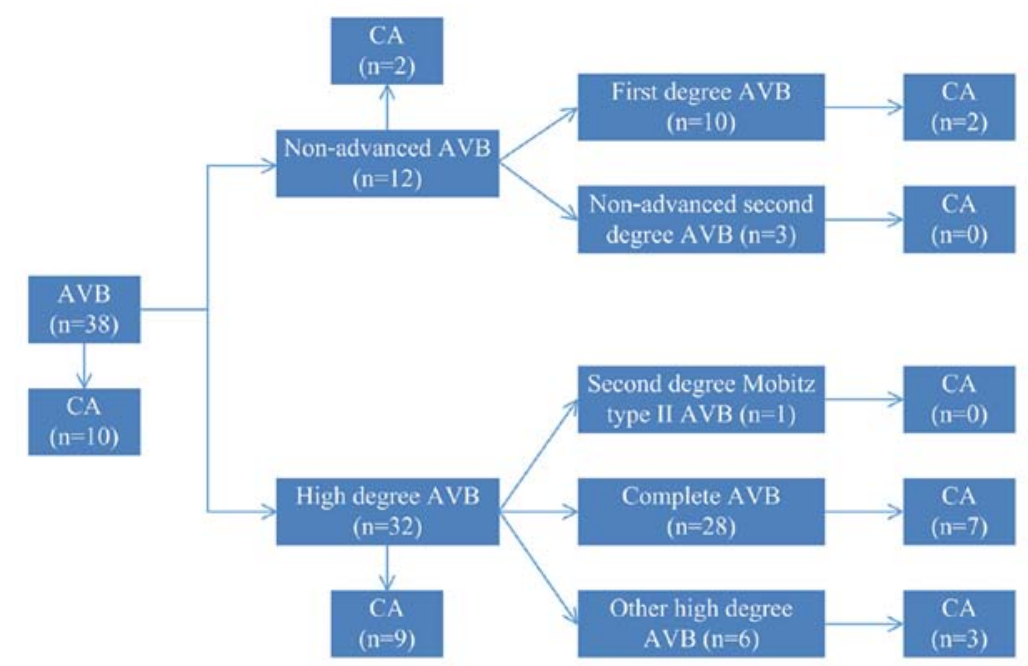

Figure 1. The incidence of CA in different types of AVB. A total of 38 patients presented with AVB including 6 patients with both non-advanced AVB and high-degree AVB. Of the aforementioned 6 patients, there was 1 case with CA. Hence, 12 cases had non-advanced AVB, including 2 cases with CA; and 32 cases had high-degree AVB, including 9 cases with CA. Of the aforementioned 12 patients, 1 case had both first-degree AVB and non-advanced second-degree AVB; of the aforementioned 32 patients, 3 cases had both complete AVB and other high-degree AVB, including 1 case with CA. AVB, atrioventricular block; CA, cardiac arrest.

failure associated with the transition of ECG changes to fatal arrhythmia, the evaluation of patients with myocarditis is a key clinical dilemma (2,3,19). ECG is a non-invasive, quick, safe and widely used screening tool that has a crucial role in diagnostics 


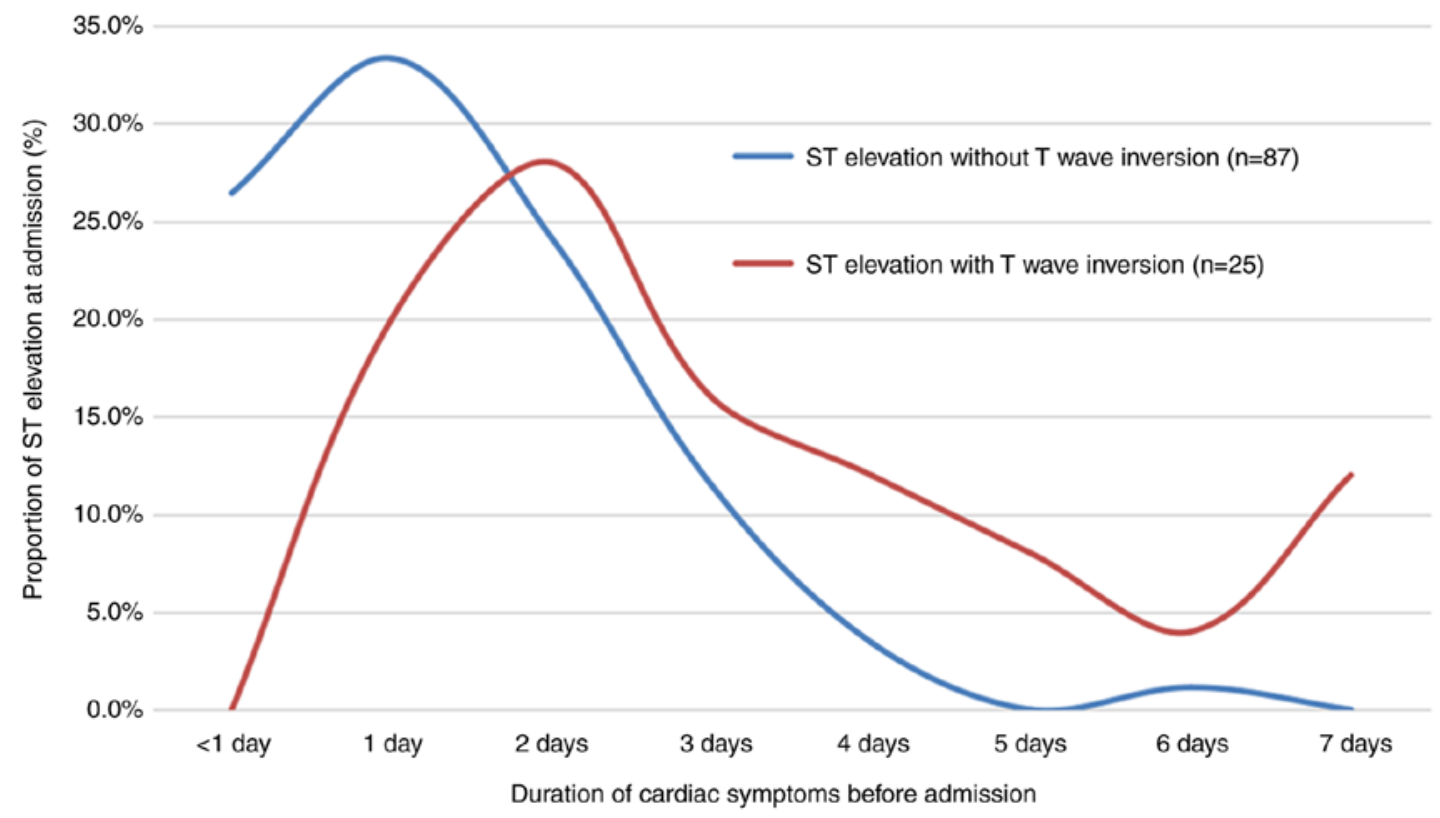

Figure 2. Time distribution of cardiac symptoms prior to admission for ST elevation at admission. A total of 112 patients with myocarditis displayed ST elevation on admission. Of these, 87 had ST elevation without T-wave inversion and the median duration of cardiac symptoms prior to admission was 1.5 days. The remaining 25 patients had ST elevation with T-wave inversion and the median duration of cardiac symptoms prior to admission was 3.1 days. ST elevation without T-wave inversion was associated with a shorter duration of cardiac symptoms (median values, 1.5 vs. 3.1 days; $\mathrm{P}<0.001$ ) compared with ST elevation with T-wave inversion.

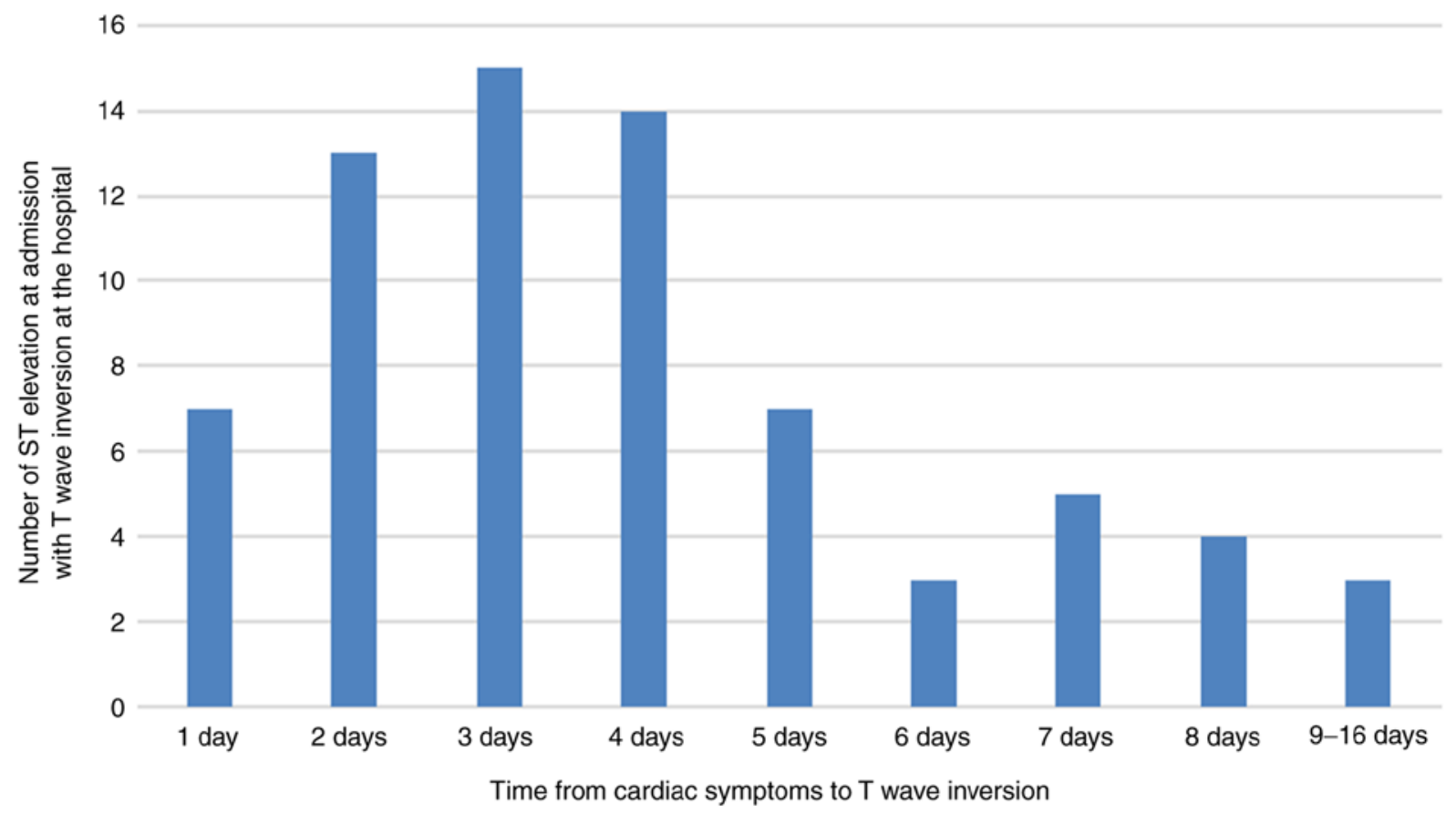

Figure 3. Time of T-wave inversion for ST elevation on admission. Of the patients with myocarditis who displayed ST elevation without T-wave inversion on admission, 71 patients $(81.6 \%$ ) had T-wave inversions at the hospital. The median time from the onset of cardiac symptoms to T-wave inversion was 4.0 days (range, 1.0-16.0 days).

and differential diagnostics, assessments of severity, determinations of patient prognosis and analyses of the disease course. To the best of our knowledge, the present case series of 274 patients is the largest study to date describing ECG findings for acute myocarditis, ECG findings associated with FM and the characteristics of ST elevation at admission. In patients with myocarditis, the occurrence rate of ECG changes ranges from 88.2 to $100 \%$, and various ECG abnormalities may exist (4-6,15). In the present study, univariate analysis demonstrated that 12 ECG parameters were associated with FM. Furthermore, multivariate logistic regression analysis revealed that 5 of these factors were independent predictive factors for FM. These independent predictive factors were VT, high-degree AVB, sinus tachycardia, low voltage and QRS duration of $\geq 120 \mathrm{msec}$. 

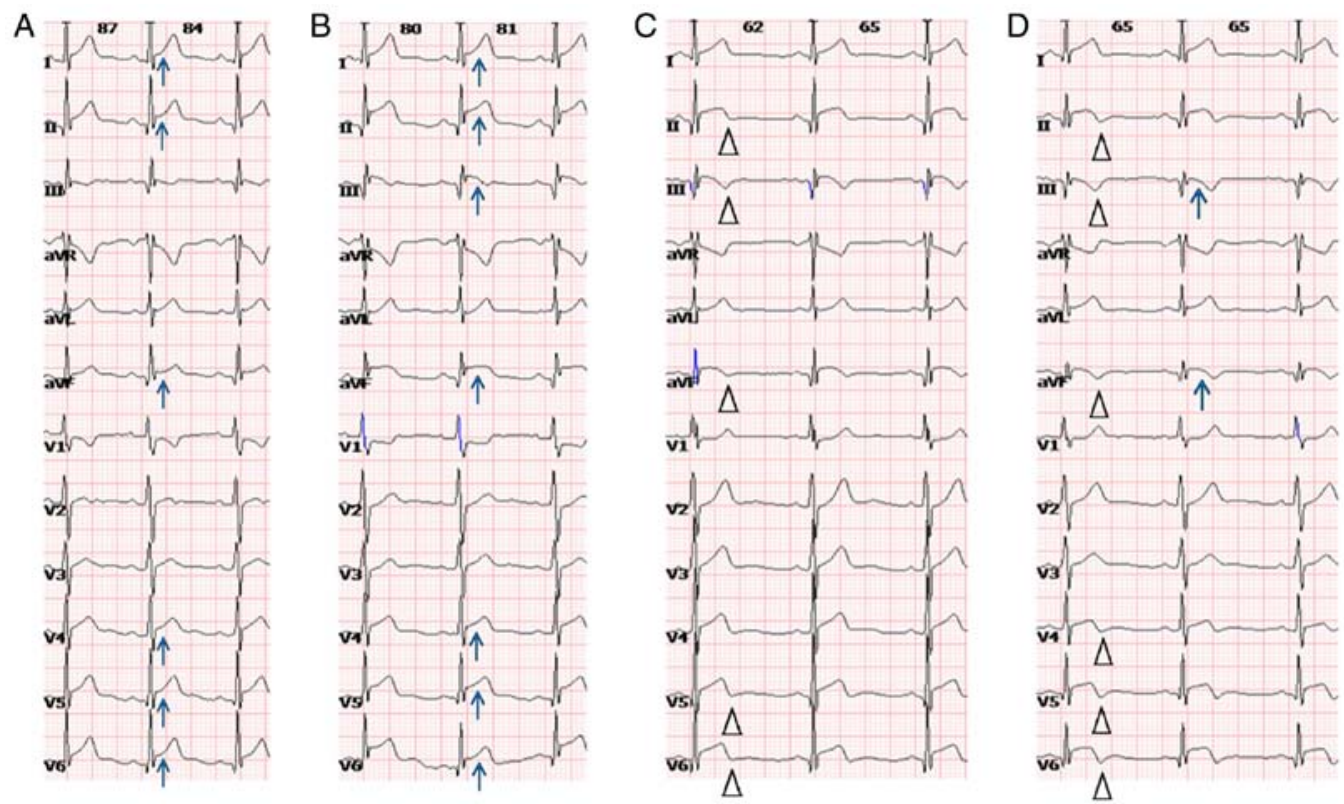

Figure 4. Evolution from ST elevation at admission to T-wave inversion in a 14-year-old male patient. (A) ECG on admission indicated ST elevation in leads I, II, avF and V4-V6 (arrow) (0.5 days from the onset of cardiac symptoms). (B) At one day after admission, the ST elevation (arrow) on ECG was higher compared with that on the previous ECG. (C) On the second day after admission, the ECG exhibited a slight inversion of T-waves in leads II, III, avF and V5-V6 (triangle), and the ST elevation was lower compared with that in the previous ECG. (D) On the third day after admission, the ECG indicated an obvious inversion of T-waves in leads II, III, avF and V4-V6 (triangle), and the ST elevation was lower compared with that in the previous ECG. In addition, the ST elevation in leads III and avF had almost disappeared (arrow). ECG, electrocardiogram.

VT is a common tachyarrhythmia that frequently occurs in viral myocarditis (19). The present study indicated that VT accounted for $6.2 \%$ and was an independent predictive factor of FM. Miyake et al (5) demonstrated that arrhythmias (70\% VF) were associated with FM and poor outcomes. This was in accordance with the present study. The mechanisms underlying arrhythmias may be associated with transient inflammatory-cell infiltration of the myocardium, oedema of myocardial cells and the intramyocardial cellular space, myocardial fibrosis, ion-channel dysfunction and ischemia $(5,11,19)$. Sinus tachycardia was the most common arrhythmia, a typical finding of tachyarrhythmias (11). In the present study, sinus tachycardia, accounting for $26.3 \%$, was also an independent predictive factor of FM. Cardiogenic shock, occurring in 71/77 of FM cases in the present study, was the most common symptom of FM, which may explain why sinus tachycardia is an independent predictive factor.

High-degree AVB/complete AVB, with an incidence ranging from 1.1 to $14.8 \%$, has been associated with the severity of the disease, which is similar to the results of the present study $(6,7,14)$. The present study indicated that high-degree AVB was an independent predictive factor of FM and the incidence rate of CA (7.2 vs. $28.1 \%$; $\mathrm{P}=0.001)$ was different between patients with non-AVB and high-degree AVB. This result was higher compared with the incidence rate of $\mathrm{CA}$ determined by Ogunbayo et al (7), who reported a difference between patients with non-heart block and high-degree AVB (CA incidence of 1.7 vs. $17.4 \%$, respectively; $\mathrm{P}<0.001$ ). In addition, the present study indicated that 6 patients had both non-advanced AVB and high-degree AVB, which indicated that high-degree AVB and non-advanced AVB may be present in parallel as the disease progresses. In 27 cases of CA, the initial rhythm was shockable (VT/VF) in 21 patients (77.8\%).
Similarly, according to recent guidelines, AMI-induced CA also has a high rate of shockable rhythms as the first rhythms (19). Survival statistics appear to be better when the first rhythms are identified as shockable compared with pulseless electrical activity or asystole (20). For secondary prevention, the 2017 guidelines recommend that an implantable cardioverter defibrillator is mandatory after VF or VT that is not due to reversible causes (19). Pavlicek et al (21) revealed that half of patients with suspected myocarditis experienced VF or VT after implantable cardioverter defibrillator therapy.

Low voltage is a common ECG abnormality in myocarditis and is associated with the severity of the disease. Casadonte et al (14) examined paediatric patients with FM and indicated that low voltage was present in 16 of 27 patients. Lee et al (6) studied patients with histologically confirmed lymphocytic myocarditis and indicated that low voltage was present in 28 of 35 patients. Miyake et al (5) studied patients $\leq 21$ years hospitalized with acute myocarditis between 1996 and 2012 and demonstrated that low voltage, detected in 81 of 85 patients, was associated with the occurrence of clinically significant arrhythmias. In the large cohort of patients in the present study, low voltage was present in 44 of 274 patients and was associated with FM. The multivariate analysis further verified that low voltage was an independent predictive factor of FM. Although low voltage is frequently encountered in myocarditis, the pathophysiology remains elusive. Myocardial oedema may be an explanation for low voltage (5). Furthermore, the present study suggested that a QRS duration of $\geq 120 \mathrm{msec}$ (including left $\mathrm{BBB}$, right $\mathrm{BBB}$ and intraventricular block) was an independent predictive factor of FM. Ukena et al (8) studied patients with clinically suspected acute myocarditis between 1995 and 2009 and determined that a QRS duration of $\geq 120 \mathrm{msec}$ (left BBB, right BBB and indeterminate BBB) 
was an independent predictor of cardiac death or heart transplantation. Morgera et al (22) indicated that adult myocarditis presenting with left BBB was associated with unfavourable outcome. Miyake et al (5) suggested that in paediatric myocarditis, left BBB increased the risk of poor outcome by 7.8-fold. These results suggested that QRS prolongation was an independent factor for predicting poor outcomes, which was corroborated by the results of the present study.

As mentioned above, ECG has a key role in the assessment of severity. Of note, UCG, whose diagnostic criteria have been suggested and adopted for myocarditis patients, may also be used to distinguish FM from myocarditis $(23,24)$. In the near future, the role of UCG in myocarditis patients we be further studied. Generally speaking, compared with UCG assessment, ECG is more rapid, timely and convenient. The ECG may detect certain dynamic changes over time, which are meaningful and helpful in guiding treatments, including cardioversion, defibrillation and pacemaker implantation.

Undoubtedly, T-wave inversion and ST elevation are the most common and the most widely studied ECG changes in myocarditis, both of which are types of ST-T-wave changes $(2,5,14,15)$. The present study suggested that T-wave inversion $(48.2 \%)$ was not associated with FM, which was similar to the results of the study by Ukena et al (8), which indicated that T-wave inversion, documented in one-third of all patients, was the most frequently observed ECG abnormality and was not predictive of cardiac death or heart transplantation. Most of the patients with myocarditis presented with ST elevation in partial leads, mimicking ECG alterations caused by AMI (4). Due to the lack of a relevant systematic study, differentiating between myocarditis and AMI presents a clinical dilemma (1). In a small study of 11 cases of myocarditis, Nakashima et al (4) observed ST elevation in 8 patients, of which only one exhibited reciprocal ST depression. These results are in line with the present findings, which are based on a larger cohort of patients. The present study indicated that 121 patients presented with ST elevation (of which 5 patients had reciprocal ST depression), and 112 patients displayed ST elevation at admission (of which 3 patients had reciprocal ST depression). Therefore, it is generally accepted that in a young patient with flu-like prodrome, ST elevation without reciprocal ST depression may be used to distinguish AMI to avoid unreasonable treatments $(1,4)$. Furthermore, the present study indicated that the FM group had significantly higher ST elevation compared with the non-FM group. However, multivariate analysis did not reveal that ST elevation was an independent predictive factor. Miyake et al (5) suggested that ST segment changes in ECG substantially increased the risk by raising subacute arrhythmias. Nakashima et al (4) mentioned that severe inflammatory changes in the myocardium and/or pericardial involvement may contribute to ST elevation. ST segment changes may reflect ongoing myocardial oedema that leads to worsening of cell membrane leakage, the accumulation of bioproducts and a decrease in energy delivery and oxygenation of myocardial tissue (5).

Of note, ST-T-wave changes in patients with myocarditis evolve as the disease progresses. Certain patients with ST elevation return to normal over several days, while others progress further to T-wave inversion (11). The present study indicated that of 87 patients with ST elevation without T-wave inversions at admission, 71 (81.6\%) had T-wave inversions at the hospital. This rate was higher compared with that determined by Wu et al (1), who reported on 18 cases of ST elevation without T-wave inversion at admission, of which 7 cases had achieved T-wave inversion at discharge. The present results were in line with the previous observation that the number of leads with ST elevation, from the acute to the convalescent stage, was notably decreased and was frequently replaced by negative $\mathrm{T}$ waves (4). In addition, the present study further indicated that the time of T-wave inversion since the initial cardiac symptom onset was 4.0 days. Finally, the present study comprised 25 patients that had ST elevation with T-wave inversion at admission, not including the 87 patients with ST elevation without T-wave inversion. ST elevation without T-wave inversion was associated with a lower duration of cardiac symptoms compared with ST elevation with T-wave inversion (1.5 vs. 3.1 days; $\mathrm{P}<0.001)$. These data may help to analyse the course of disease development.

In conclusion, acute myocarditis is a common disease with high mortality and various dynamic changes in the ECG. ECG should be widely used to assess disease severity and for the analysis of the characteristics of ST elevation at admission. The present data will provide an important reference for managing patients with suspected myocarditis.

\section{Acknowledgements}

Not applicable.

\section{Funding}

No funding was received.

\section{Availability of data and materials}

The datasets used and/or analyzed during the current study are available from the corresponding author on reasonable request.

\section{Authors' contributions}

JC, SC, ZL, WH and YL were involved in the conception and design of the study. JC, PZ, HW, JS, YN and LL collected the data. JC, HW and LL analyzed the ECG. JC and YL drafted the manuscript. JC, PZ, JS, YN and YL analyzed and interpreted the data and performed the statistical analysis. SC, ZL, WH and YL were major contributors in writing and revising the manuscript. All authors read and approved the final manuscript.

\section{Ethics approval and consent to participate}

The study was approved by the ethics committee of The First Affiliated Hospital of Wenzhou Medical University (approval no. KY2020-103) and Wenzhou People's Hospital (approval no. 2020-96).

\section{Patient consent for publication}

Not applicable. 


\section{Competing interests}

The authors declare that they have no competing interests.

\section{References}

1. Wu S, Yang YM, Zhu J, Wan HB, Wang J, Zhang H and Shao XH: Clinical characteristics and outcomes of patients with myocarditis mimicking ST-segment elevation myocardial infarction: Analysis of a case series. Medicine (Baltimore) 96: e6863, 2017.

2. Caforio AL, Pankuweit S, Arbustini E, Basso C, Gimeno-Blanes J, Felix SB, Fu M, Heliö T, Heymans S, Jahns R, et al: Current state of knowledge on aetiology, diagnosis, management, and therapy of myocarditis: A position statement of the European society of cardiology working group on myocardial and pericardial diseases. Eur Heart J 34: 2636-2648, 2648a-2648d, 2013.

3. Biesbroek PS, Beek AM, Germans T, Niessen HW and van Rossum AC: Diagnosis of myocarditis: Current state and future perspectives. Int J Cardiol 191: 211-219, 2015.

4. Nakashima H, Honda Y and Katayama T: Serial electrocardiographic findings in acute myocarditis. Intern Med 33: 659-666, 1994.

5. Miyake CY, Teele SA, Chen L, Motonaga KS, Dubin AM, Balasubramanian S, Balise RR, Rosenthal DN, Alexander ME, Walsh EP and Mah DY: In-hospital arrhythmia development and outcomes in pediatric patients with acute myocarditis. Am J Cardiol 113: 535-540, 2014

6. Lee KJ, McCrindle BW, Bohn DJ, Wilson GJ, Taylor GP, Freedom RM, Smallhorn JF and Benson LN: Clinical outcomes of acute myocarditis in childhood. Heart 82: 226-233, 1999.

7. Ogunbayo GO, Elayi SC, Ha LD, Olorunfemi O, Elbadawi A, Saheed D and Sorrell VL: Outcomes of heart block in myocarditis: A review of 31,760 Patients. Heart Lung Circ 28: 272-276, 2019.

8. Ukena C, Mahfoud F, Kindermann I, Kandolf R, Kindermann M and Bohm M: Prognostic electrocardiographic parameters in patients with suspected myocarditis. Eur J Heart Fail 13: 398-405, 2011.

9. Veronese G, Ammirati E, Cipriani M and Frigerio M: Fulminant myocarditis: Characteristics, treatment, and outcomes. Anatol J Cardiol 19: 279-286, 2018.

10. JCS Joint Working Group: Guidelines for diagnosis and treatment of myocarditis (JCS 2009): Digest version. Circ J 75: 734-743, 2011.

11. Baksi AJ, Kanaganayagam GS and Prasad SK: Arrhythmias in viral myocarditis and pericarditis. Card Electrophysiol Clin 7: 269-281, 2015.

12. Ichikawa R, Sumitomo N, Komori A, Abe Y, Nakamura T, Fukuhara J, Matsumura M, Miyashita M, Kanamaru H, Ayusawa $\mathrm{M}$ and Mugishima $\mathrm{H}$ : The follow-up evaluation of electrocardiogram and arrhythmias in children with fulminant myocarditis. Circ J 75: 932-938, 2011.

13. Aretz HT, Billingham ME, Edwards WD, Factor SM, Fallon JT, Fenoglio JJ Jr, Olsen EG and Schoen FJ: Myocarditis. A histopathologic definition and classification. Am J Cardiovasc Pathol 1: 3-14, 1987.

14. Casadonte JR, Mazwi ML, Gambetta KE, Palac HL, McBride ME, Eltayeb OM, Monge MC, Backer CL and Costello JM: Risk factors for cardiac arrest or mechanical circulatory support in children with fulminant myocarditis. Pediatr Cardiol 38: 128-134, 2017.

15. Ammirati E, Cipriani M, Lilliu M, Sormani P, Varrenti M, Raineri C, Petrella D, Garascia A, Pedrotti P, Roghi A, et al: Survival and left ventricular function changes in fulminant versus nonfulminant acute myocarditis. Circulation 136: 529-545, 2017.
16. Kligfield P, Gettes LS, Bailey JJ, Childers R, Deal BJ, Hancock EW, van Herpen G, Kors JA, Macfarlane P, Mirvis DM, et al: Recommendations for the standardization and interpretation of the electrocardiogram: Part I: The electrocardiogram and its technology a scientific statement from the American heart association electrocardiography and arrhythmias committee, council on clinical cardiology; the American college of cardiology foundation; and the heart rhythm society endorsed by the international society for computerized electrocardiology. J Am Coll Cardiol 49: 1109-1127, 2007.

17. Lei YL, Chen SQ, Li ZP, Xue JK, Wang TT, Zhou ZL, Xu HQ, Lu YR, Li HP, Huang WJ and Cheng JT: Percutaneous coronary intervention improved outcomes of cardiac arrest after acute myocardial infarction: A 2-year study. Int J Clin Exp Med 10: 3097-3105, 2017

18. Rautaharju PM, Surawicz B, Gettes LS, Bailey JJ, Childers R, Deal BJ, Gorgels A, Hancock EW, Josephson M, Kligfield P, et al: AHA/ACCF/HRS recommendations for the standardization and interpretation of the electrocardiogram: Part IV: The ST segment, T and U waves, and the QT interval: a scientific statement from the American heart association electrocardiography and arrhythmias committee, council on clinical cardiology; the american college of cardiology foundation; and the heart rhythm society. Endorsed by the international society for computerized electrocardiology. J Am Coll Cardiol 53: 982-991, 2009.

19. Al-Khatib SM, Stevenson WG, Ackerman MJ, Bryant WJ, Callans DJ, Curtis AB, Deal BJ, Dickfeld T, Field ME, Fonarow GC, et al: 2017 AHA/ACC/HRS guideline for management of patients with ventricular arrhythmias and the prevention of Sudden cardiac death: A Report of the American college of cardiology/American heart association task force on clinical practice guidelines and the heart rhythm society. Circulation 138: e272-e391, 2018.

20. Myerburg RJ, Halperin H, Egan DA, Boineau R, Chugh SS, Gillis AM, Goldhaber JI, Lathrop DA, Liu P, Niemann JT, et al: Pulseless electric activity: Definition, causes, mechanisms, management, and research priorities for the next decade: Report from a national heart, lung, and blood institute workshop. Circulation 128: 2532-2541, 2013.

21. Pavlicek V, Kindermann I, Wintrich J, Mahfoud F, Klingel K, Böhm M and Ukena C: Ventricular arrhythmias and myocardial inflammation: Long-term follow-up of patients with suspected myocarditis. Int J Cardiol 274: 132-137, 2019.

22. Morgera T, Di Lenarda A, Dreas L, Pinamonti B, Humar F, Bussani R, Silvestri F, Chersevani D and Camerini F: Electrocardiography of myocarditis revisited: Clinical and prognostic significance of electrocardiographic changes. Am Heart J 124: 455-467, 1992.

23. Matshela MR: The role of echocardiography in acute viral myocarditis. Cardiovasc J Afr 30: 239-244, 2019.

24. Chang YJ, Hsiao HJ, Hsia SH, Lin JJ, Hwang MS, Chung HT, Chen CL, Huang YC and Tsai MH: Analysis of clinical parameters and echocardiography as predictors of fatal pediatric myocarditis. PLoS One 14: e0214087, 2019.

This work is licensed under a Creative Commons Attribution-NonCommercial-NoDerivatives 4.0 International (CC BY-NC-ND 4.0) License. 